\title{
The Adjustment of Surface Wind and Pressure by Sasaki's Variational Matching Technique
}

\author{
John M. Lewis and Thomas H. Grayson ${ }^{1}$ \\ Fleet Numerical Weather Central, Monterey, Calif. 93940 \\ (Manuscript received 28 October 1971, in revised form 26 January 1972)
}

\begin{abstract}
The sea level pressure and surface wind fields operationally produced at Fleet Numerical Weather Central are adjusted by using numerical variational analysis. The analysis region is a global band extending from $40 \mathrm{~S}$ to $60 \mathrm{~N}$ and the successive corrections method is used to generate the initial or input fields. These fields are then adjusted within the framework of the variational method by requiring that they satisfy certain governing dynamical equations.

A detailed study of this method is made in the Atlantic Ocean region on 4 January 1971. There is convincing evidence that small-scale wind information is incorporated into the pressure field and that the adjusted wind field has been modified to account for ageostrophic motion.
\end{abstract}

\section{Introduction}

Surface analyses of wind and sea level pressure are operationally produced every $6 \mathrm{hr}$ on a global band grid at Fleet Numerical Weather Central (FNWC). This grid encompasses the latitudinal band between $40 \mathrm{~S}$ and $60 \mathrm{~N}$ and is mapped on a Mercator secant projection true at $22.5 \mathrm{~N}$ and $22.5 \mathrm{~S}$. The mesh length varies from $150 \mathrm{n} \mathrm{mi}$ at the equator to $75 \mathrm{n} \mathrm{mi}$ at $60 \mathrm{~N}$. Besides providing diagnostic analyses to the outlying weather centrals, these products are used as input to forecast models of wind-generated waves and as guides to the Optimum Track Ship Routing (OTSR) group at FNWC.

The successive corrections method (SCM) described by Cressman (1959) is the basic analysis procedure used to obtain both the wind and pressure. The details of the FNWC version of this technique have been discussed by Grayson (1971). We briefly review the main features of this analysis procedure. First, the pressure analysis is routinely accomplished using the six-hour-old field as a guess. This guess is then successively corrected to accommodate the current pressure observations. Three scans are used employing radii of influence which are a function of data density. In effect, the scan radii are reduced more slowly in data-sparse regions. Maine and Seaman (1967) have used this methodology for the Australian region and Grayson has incorporated their idea into the FNWC scheme. For the purpose of our discussion we can characterize the radii as 450,300 and $150 \mathrm{n} \mathrm{mi}$, respectively:

1 Current affiliation: Techniques Development Laboratory, NOAA, Silver Spring, Md.
The wind analysis is geostrophically coupled with the pressure field outside the latitude belt $20 \mathrm{~S}-20 \mathrm{~N}$. The coupling is accomplished by using a modified geostrophic wind as a first guess for the wind field. The modification accounts for the effects of static stability near the air-ocean interface (see Hubert and Mendenhall, 1970). In the band 20S-20N, the six-hourold wind analysis, i.e., persistence, is used. The wind analysis is accomplished in two scans with influence radii of $300 \mathrm{n} \mathrm{mi}$ and $150 \mathrm{n} \mathrm{mi}$, respectively.

A major deficiency of this procedure is the inability to incorporate wind reports into the pressure analysis. The idea of creating a pressure report from a wind observation, using the geostrophic relation, has been extensively used in mid-latitudes (see Gandin, 1965). Using this approach outside an equatorial belt would be consistent with the guess for the wind. Nevertheless, rigid adherence to the geostrophic relation severely limits the direct application of the analysis to 1) diagnostic calculations of kinematic properties such as divergence and vorticity, and 2) initialization of prognostic models where a geostrophic bias in the fields can create unwanted modes.

From a pragmatic viewpoint, the following questions must be answered:

1) Given the operational products derived by the methodology described above, how can the fields be adjusted so that wind information can be fed into the pressure analysis in a consistent manner over the entire grid?

2) How can the pressure information be used to generate wind fields, again, in a consistent manner over the entire range of latitudes? 
In addition to answering these questions, there should be an attempt to make the adjusted analyses fit those observations which have passed the gross error checks and lateral reject checks and have subsequently been included in the conventional analysis. The analysis in a data-rich region should be anchored to representative reports but still subject to dynamical adjustment. That is, final analysis should weight observational information (in accord with data density) and bring in the governing dynamical relations to couple wind and pressure, and assure an adjustment that suppresses meteorological noise resulting from the successive corrections method of analysis.

The balance equation (see Charney, 1955) has enjoyed considerable success in the adjustment of pressure and wind fields. This diagnostic relation is well suited to the description of synoptic-scale features in mid-latitudes, but is subject to certain difficulties in the equatorial region for reasons related to convergence of iterative schemes or accuracy of the solution (Houghton and Washington, 1969). The ability to join this dynamical constraint with observations of both pressure and wind was demonstrated by Sasaki (1958). Stephens (1970) has used this approach on analytical fields to verify that the inclusion of both pressure and wind fields according to their relative accuracies yields better results than those obtained from a single field.

The proposed analysis scheme uses an approach based on Sasaki's recent extensions of his original (1958) variational formulation (see Sasaki, 1969). With these extensions, it is now possible to include the prognostic equations as constraints and incorporate data from different time periods.

The time-dependent momentum equations, including the parameterization of friction, are used as dynamical constraints in this analysis scheme. A similar set of equations has been employed by Lewis (1971) in the analysis of surface conditions accompanying severe storms. A major problem encountered in that study was the convergence of the Euler equations when the ratio of the dynamical weight to the observational weight exceeded a critical value. This critical value apparently changed the nature of the equations from an elliptic system to a hyperbolic or parabolic system. Since the boundary conditions were designed for elliptic equations (Dirichlet conditions), a unique solution was nonexistant when the critical value was exceeded and altered the type of equation. This is analogous to the mixed-type boundary value problem encountered when solving the balance equation for the streamfunction. A more detailed description of the uniqueness problem as it relates to the variational analysis can be found in Sasaki (1970a). The present formulation guarantees an elliptic system of Euler equations and succeeds in bypassing the mixed-type boundary value problem.

The numerical variational analysis (NVA) method is applied to data collected on 4 January 1971. The region of interest is centered over the Atlantic Ocean and the comparison between input or conventional analysis and NVA is made.

\section{Dynamical constraints and the variational analysis formulation}

The horizontal momentum equations will be used as the dynamical constraints in this analysis scheme. The equations are written for Mercator coordinates and assume the form:

$$
\begin{array}{r}
\frac{\partial u}{\partial t}+\mathrm{V} \cdot \nabla u-f v=-m-\frac{1}{\rho} \frac{\partial p}{\partial x}+\frac{1}{\rho} \frac{\partial \tau_{x}}{\partial z}, \\
\frac{\partial v}{\partial t}+\mathrm{V} \cdot \nabla v+f u=-m-\frac{\partial p}{\rho}+\frac{1}{\partial y} \frac{\partial \tau_{y}}{\partial z},
\end{array}
$$

where:

$\begin{array}{ll}t & \text { time } \\ x & \text { east-west coordinate (positive eastward) } \\ y & \text { north-south coordinate (positive northward) } \\ z & \text { vertical coordinate (positive upward) } \\ \mathbf{V} & \hat{u \mathbf{i}}+v \hat{\mathbf{j}} \text {, horizontal velocity } \\ \hat{\mathbf{i}} & \text { unit vector along positive } x \\ \hat{\mathbf{j}} & \text { unit vector along positive } y \\ p & \text { pressure } \\ \rho & \text { density }\left(1.22 \mathrm{~kg} \mathrm{~m}^{-3}\right) \\ f & \text { Coriolis parameter } \\ \tau & \tau_{x} \hat{\mathbf{i}}+\tau_{y} \hat{\mathbf{j}}, \text { stress } \\ m & \text { cos } \phi_{0} \sec \phi, \text { image scale factor } \\ \phi & \text { latitude }\left(\phi_{0}=22.5^{\circ}\right) \\ & \partial() \hat{\mathbf{i}}+m \frac{\partial()}{\partial y} \hat{\mathbf{j}} \text {, gradient operator } \\ \nabla & m \frac{\partial x}{}\end{array}$

The image scale factor $m$ appears naturally with the horizontal space derivatives. Consequently, we will simplify our notation and assume that any reference to $\partial / \partial x$ or $\partial / \partial y$ always implies $m \partial / \partial x$ or $m \partial / \partial y$, respectively.

The vertical advection terms are conspicuously absent from the momentum equations. These terms can be significant in strong baroclinic regions and in areas where the terrain induces vertical motion. At present, the upper air analysis over the global band grid is incomplete and vertical shear information is lacking. Eventually, the surface and upper air analyses will be unified and the dynamical terms involving the vertical transport of momentum will be included.

Since the analysis scheme has access to surface information only, the change of stress with height is impossible to calculate directly. This effect can be parameterized, however, by considering a finite-difference approximation between the surface and the top of 
the planetary boundary layer. In effect

$$
\frac{1}{\rho} \frac{\partial \tau}{\partial z}=\frac{-\tau(0)}{\bar{\rho} H}
$$

where the stress has vanished at the top of the layer of depth $H$, and $\bar{\rho}$ represents the mean density in this boundary layer. Following Lettau's (1959) arguments, we assume the stress proportional to the square of the wind speed and get

$$
\frac{1}{\rho} \frac{\partial \tau}{\partial z}=-\frac{\rho C_{D}|\mathbf{V}|}{\bar{\rho} H}(\hat{\hat{\mathbf{i}}}+v \hat{\mathbf{j}}),
$$

where $C_{D}$ is a drag coefficient. For our analysis scheme, the frictional effects will be given by

$$
\frac{1}{\rho} \frac{\partial \tau}{\partial z}=-\kappa(u \hat{\mathbf{i}}+v \hat{\mathbf{j}})
$$

where $\kappa$ is assumed constant $\left(10^{-1} \mathrm{hr}^{-1}\right)$. This parameterization is known as the Guldberg-Mohn hypothesis (Hess, 1959). The value of $\kappa$ is estimated by using the values: $|\mathbf{V}|=20 \mathrm{kt}, C_{D}=2.5 \times 10^{-3}, \rho / \bar{\rho}=1, H=0.5$ $\mathrm{n}$ mi. The drag coefficient is the mean of the wintertime land and sea values quoted by Kung (1963). The values for both the wind speed and height of the boundary layer have been chosen in accord with geostrophic scale theory. Since the analysis region covers the tropical belt which is typified by different scale values, the parameterization should eventually be modified to accommodate both mid-latitude and tropical regimes.

As mentioned in the introduction, the variational scheme will attempt to anchor the analysis to the observations in data-rich regions and provide a method of "extrapolating" from these regions into datadeficient regions. The extrapolation is possible by using the dynamical constraints, which in this particular application are the horizontal momentum equations. Conceptually, one can imagine holes, or data void regions, in the analysis space which are fastened at the edges by data. The NVA scheme provides a means of fitting the interior of these holes in a manner consistent with the dynamical equations and blending with available data.

The analysis couples observations (input analysis) and dynamics through the minimization of the functional

$$
I=\iint_{S}\left\{\begin{array}{c}
{\left[\tilde{\alpha}(u-\tilde{u})^{2}+\tilde{\alpha}(v-\tilde{v})^{2}+\tilde{\beta}(p-\tilde{p})^{2}\right]} \\
+\left[\alpha\left(\frac{\partial u}{\partial t}\right)^{2}+\alpha\left(\frac{\partial v}{\partial t}\right)^{2}\right]
\end{array}\right\} d S
$$

where $(\sim)$ indicates the conventional analysis and where the surface integral extends over the entire analysis region $S$. This formulation is called the timewise localized version of NVA (Sasaki, 1970a). The first set of bracketed terms forces the analysis toward the input fields in direct proportion to the "observational" weights on wind and pressure, $\tilde{\alpha}$ and $\widetilde{\beta}$, respectively. The "observational" weights in this study represent the weights given to the conventional analysis. These weights are specified beforehand and are not determined as a result of the variational operations. The second set of terms brings the dynamics into the analysis and controls the degree of steadiness in direct proportion to the weight $\alpha$, again specified beforehand. These weights, $\alpha, \tilde{\alpha}$ and $\tilde{\beta}$, have often been called the precision moduli of Gauss in the literature on least-squares.

The "observations" represented in (6), viz., $\tilde{u}, \tilde{v}$, and $\tilde{p}$, are dependent outside the latitude belt $20 \mathrm{~N}-20 \mathrm{~S}$ since the guess for the wind field is derived from the pressure analysis. This coupling is undesirable when used in conjunction with the variational matching. In particular, the least-squares formulation guarantees the maximum likelihood estimates only when the "observations" are independent (Cramér, 1955). The conventional wind analysis, $\tilde{u}$ and $\tilde{v}$, over data-rich regions such as the continents of North America and Europe can effectively overcome this coupling, but the sparse data regions will certainly be strongly influenced by the guess. It is clear that this geostrophic coupling should ultimately be eliminated and the variational matching should operate on independent fields.

The weights $\tilde{\alpha}$ and $\widetilde{\beta}$ are expressed in terms of the mean-square difference between input fields and NVA fields. [The terminology mean-square error and rootmean-square error (RMSE) is reserved for comparing the analyses with the actual observations in the section on verification.] By analogy, the weight $\alpha$ can be specified in terms of the mean-square value of the acceleration as found from (1) and (2). Using this concept the weights are given by

$$
\tilde{\alpha}=\frac{1}{\sigma_{w}}, \quad \tilde{\beta}=\frac{1}{\sigma_{p}}, \quad \alpha=\frac{1}{\sigma_{t}},
$$

where the notation is defined as follows: (i) $\sigma_{w}$ is the mean-square difference between the separate wind components of the NVA and conventional analyses, (ii) $\sigma_{p}$ is the mean-square difference between the NVA and conventional pressure fields, and (iii) $\sigma_{t}$ is the meansquare acceleration of each component calculated from the momentum equations.

These weights can be chosen to reflect the confidence in "observations" (specification $\tilde{\alpha}$ and $\widetilde{\beta}$ ) and dynamics (specification of $\alpha$ ). For example, assume that $a, b$ and $c$ denote values of $\sigma_{w}, \sigma_{p}$ and $\sigma_{t}$ which are chosen a priori. The solution of the minimization problem only guarantees that the ratios between the mean-square values are preserved. That is, the values of $\sigma_{w}, \sigma_{p}$ and $\sigma_{t}$ calculated from the solution are not necessarily equal to $a, b$ and $c$. However, they exhibit the prescribed ratios $\left(\sigma_{w}: \sigma_{p}: \sigma_{t}=a: b: c\right)$. It is sufficient to specify two 
independent ratios, such as $\tilde{\alpha} / \tilde{\beta}$ and $\alpha / \tilde{\beta}$, rather than the three weights separately.

We have invoked the following premise in specifying the weights $\tilde{\alpha}$ and $\widetilde{\beta}$ : the mean-square differences $\sigma_{w}$ and $\sigma_{p}$ decrease as the data density increases. The data density is determined from: 1) the number of accepted observations on the first pass of the SCM and 2) the distance of each of these observations from the grid point in question. Since the SCM uses distancedependent weighting, a cumulative weight is found at each grid point by totaling the individual weights from accepted observations. This cumulative weight or index of data density is used to assign $\tilde{\alpha}$ and $\widetilde{\beta}$ to grid points. In the operational version of NVA we plan to have a sliding scale for $\tilde{\alpha}$ and $\widetilde{\beta}$ which will allow them to vary in direct proportion to the data density index. To avoid unrealistically high weights in data-rich regions, the index should include a factor which accounts for the intercorrelations of data in these regions. Bergthórsson and Döös (1955) accomplished this by modifying the distance-dependent weight in the SCM. We plan to adapt this idea to our scheme.

Minimization of (6) subject to the constraints is realized by substituting $\partial u / \partial t$ and $\partial v / \partial t$ from (1) and (2) into (6), and then requiring the first variation of $I$ to vanish, i.e.,

$$
\delta I=0 \text {, }
$$

where $\delta$ is the variational operator. Although somewhat tedious, the manipulation and mechanics of obtaining the governing Euler equations is straightforward. These governing analysis equations (three simultaneous second-order partial differential equations) were then applied to our test case.

The difficulty in obtaining convergent solutions for certain ratios of the observational weights to the dynamic weight persisted as previously mentioned in connection with the work of Lewis (1971). Since we felt that a wide range of weights was necessary for operational application on the global band grid, we decided to modify the dynamical constraints to insure an elliptic system which would be free from these problems of convergence. This modification amounts to changing the nonlinear terms in both (1) and (2). In particular, we replace $V \cdot \nabla u$ by $\tilde{V} \cdot \nabla \tilde{u}$ and $V \cdot \nabla v$ by $\tilde{\mathbf{V}} \cdot \nabla \tilde{v}$. Thus, the nonlinear terms are approximated by the input fields and become pre-specified functions of space. Subsequent to the completion of our research, we became aware that Stephens (1966) had replaced the nonlinear advection terms by observations so that he could handle these difficult terms.

We have undoubtedly introduced some error into the constraints (1) and (2), especially in data-deficient regions. Nevertheless, the sense and order of magnitude of these nonlinear terms are generally correct. Indeed, our case study shows beneficial effects which are directly attributable to this formulation. From an operational viewpoint, the added flexibility in specifica- tion of the weights justifies the imperfections we have introduced in the constraints.

In order to directly incorporate the assumptions on parameterization of friction and the special handling of the nonlinear terms, the momentum equations are rewritten as

$$
\begin{gathered}
\frac{\partial u}{\partial t}+\tilde{\mathbf{V}} \cdot \nabla \tilde{u}-f v=-\frac{1}{\rho} \frac{\partial p}{\partial x}-\kappa u, \\
\frac{\partial v}{\partial t}+\tilde{\mathbf{V}} \cdot \nabla \tilde{v}+f u=-\frac{1}{\rho} \frac{\partial p}{\partial y}-\kappa v .
\end{gathered}
$$

\section{The Euler equations}

Using the modified momentum equations (9) and (10) as the dynamic constraints and substitution into (8) leads to the relation

$$
0=\iint_{s}(U \delta u+V \delta v+P \delta p) d s+\text { boundary terms, }
$$

where $\delta u, \delta v$ and $\delta p$ are the variations of the two velocity components and the pressure, respectively. This form of the integral relation is obtained by using integration by parts, the standard procedure in the calculus of variations. The expressions $U, V$ and $P$ are partial differential equations involving $u, v$ and $p$; however, they are free from any terms involving $\delta u, \delta v$ or $\delta p$.

When integrating by parts to reduce the integrand to the form in (11), a set of terms to be evaluated at the boundaries appears. These terms constitute the so-called natural boundary conditions accompanying all variational calculus problems. For our particular application, we require $u, v$ and $p$ to be equal to the input values at the boundaries; in effect, we impose Dirichlet conditions and they cause the boundary terms to vanish. A concise and extremely lucid account of these terms in physical science problems can be found in Lanczos (1970).

Since the variations of $u, v$ and $p$ are independent, the solution to (11) reduces to

$$
U=0, \quad V=0, \quad P=0 .
$$

These are the Euler equations for our particular variational formulation; the differential expressions are as follows:

$$
\begin{gathered}
U=\left\{\begin{array}{c}
\left.\tilde{\alpha}(u-\tilde{u})+f^{2} \alpha u+\frac{\alpha f}{\rho} \frac{\partial p}{\partial y}+\frac{\alpha \kappa}{\rho} \frac{\partial p}{\partial x}\right\}=0, \\
+\kappa^{2} \alpha u+\alpha \kappa \widetilde{A}+\alpha f \widetilde{B}
\end{array}\right\} \\
V=\left\{\begin{array}{c}
\tilde{\alpha}(v-\tilde{v})+f^{2} \alpha v-\frac{\alpha f}{\rho} \frac{\partial p}{\partial x}+\frac{\alpha \kappa}{\rho} \frac{\partial p}{\partial y} \\
+\kappa^{2} \alpha v-\alpha f \widetilde{A}+\alpha \kappa \widetilde{B}
\end{array}\right\}=0,
\end{gathered}
$$




$$
P=\left\{\begin{array}{c}
\tilde{\beta}(p-\tilde{p})+\frac{\partial}{\partial x}\left(\frac{f \alpha v}{\rho}\right)-\frac{\partial}{\partial y}\left(\frac{f \alpha u}{\rho}\right) \\
-\frac{1}{\rho^{2}} \frac{\partial}{\partial x}\left(\alpha \frac{\partial p}{\partial x}\right)-\frac{1}{\rho^{2}} \frac{\partial}{\partial y}\left(\alpha \frac{\partial p}{\partial y}\right) \\
-\frac{1}{\rho} \frac{\partial}{\partial x}(\kappa \alpha u)-\frac{1}{\rho} \frac{\partial}{\partial y}(\kappa \alpha v) \\
-\frac{1}{\rho} \frac{\partial}{\partial x}(\alpha \widetilde{A})-\frac{1}{\rho} \frac{\partial}{\partial y}(\alpha \widetilde{B})
\end{array}\right\}=0,
$$

where

$$
\begin{aligned}
& \tilde{A}=\tilde{\boldsymbol{V}} \cdot \nabla \tilde{u}, \\
& \tilde{B}=\tilde{\boldsymbol{V}} \cdot \nabla \tilde{\nu} .
\end{aligned}
$$

Eqs. (13) and (14) can be solved for $u$ and $v$ as functions of $p$, respectively. These expressions are then substituted into (15) and we get the following elliptic equation (Helmholtz type) for $p$ :

$$
\nabla \cdot(r \nabla p)-\rho^{2} \tilde{\beta} p=\left\{\begin{array}{l}
-\rho^{2} \tilde{\beta} \tilde{p}+\hat{\mathbf{k}} \cdot \rho \nabla \times(f r \tilde{\mathbf{V}}) \\
-\rho \nabla \cdot(\kappa r \tilde{\mathbf{V}})-\rho \nabla \cdot(r \mathbf{C})
\end{array}\right\},
$$

where $\hat{\mathbf{k}}$ is the local vertical unit vector and vector products have been introduced for ease of notation along with the symbols

$$
\begin{gathered}
r=\frac{\alpha \tilde{\alpha}}{\tilde{\alpha}+\alpha\left(f^{2}+\kappa^{2}\right)}=\frac{\alpha}{1+(\alpha / \tilde{\alpha})\left(f^{2}+\kappa^{2}\right)}, \\
\mathbf{C}=\widetilde{A} \hat{\mathbf{i}}+\widetilde{B} \hat{\mathbf{j}} .
\end{gathered}
$$

It is interesting to note that the wind information appears with a weight $r$, which is the combination of the dynamic weight $\alpha$ and the observational weight $\tilde{\alpha}$. If $\alpha$ increases while $\bar{\alpha}$ remains constant, then $r$ increases. Similarly, if $\tilde{\alpha}$ increases while $\alpha$ remains constant, then $r$ also increases. Effectively, an increased confidence in the input winds (increase $\tilde{\alpha}$ ) or increasing the steadiness of the wind field (increase $\alpha$ ) incorporate more wind information in the pressure analysis. The critical ratio for the solution of (18) is obviously $\tilde{\beta} / r$. As this ratio increases, the NVA pressure is forced toward the input pressure and as the ratio decreases, the NVA pressure is driven toward the wind information.

Once the solution for (18) is found, it is then a simple algebraic problem to substitute this solution into (13) and (14) to obtain $u$ and $v$. It is instructive to write (13) and (14) in the form

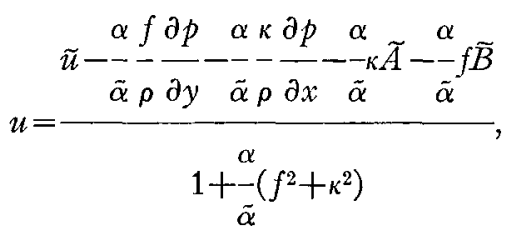

$$
v=\frac{\tilde{v}+\frac{\alpha f}{\tilde{\alpha} \rho} \frac{\partial p}{\partial x}-\frac{\alpha \kappa}{\tilde{\alpha} \rho} \rho \frac{\partial p}{\partial y}+\frac{\alpha}{\tilde{\alpha}} \tilde{A}-\frac{\alpha}{\tilde{\alpha}} \tilde{B}}{1+\frac{\alpha}{\tilde{\alpha}}\left(f^{2}+\kappa^{2}\right)} .
$$

We immediately notice that as $\alpha / \tilde{\alpha} \ll 1$, i.e., wind input has great reliability relative to the dynamical constraints, the NVA wind approaches the input winds as we would expect. We must use caution, however, in arguments related to the weighting explicitly represented in (21) and (22) since the solution for $p$ has the weights $\alpha, \tilde{\alpha}$ and $\tilde{\beta}$ implicitly represented.

The first term in both (21) and (22) incorporates the input wind in direct proportion to $\tilde{\alpha}$. The second term is a "geostrophic" contribution weighted proportional to $\alpha f$. It must be emphasized that this contribution is from the solution to (18) and is not the geostrophic wind from the input field. Term three is the frictional effect which basically produces cross-contour flow from high to low pressure in direct proportion to $k \alpha$. Finally, the last two terms stem from the nonlinear advection and account for the effects such as curvature and confluence in the wind field'.

Examination of the forcing function (the right-hand side) in (18) shows the four contributors to the solution of this Helmholtz equation. The first term counts the pressure input in direct proportion to $\widetilde{\beta}$. The second term is the vorticity of the input winds and is included in direct proportion to $r$. The third term is the divergence of the input winds and appears in proportion to the friction parameter $\kappa$. The contribution to the pressure by this term is

or

$$
\delta(\nabla \cdot r \nabla p) \approx-\rho \nabla \cdot(\kappa r \tilde{\mathbf{V}}),
$$

$$
\delta\left(\begin{array}{l}
1 \\
-\nabla p
\end{array}\right) \approx-\kappa \tilde{\mathrm{V}}
$$

which implies a pressure adjustment in response to the frictional term of the ageostrophic flow. This third term is very noisy, i.e., tends to exhibit "checkerboard" patterns on the synoptic-scale charts. This is a well known fact and the reason is unquestionably related to the insufficient accuracy of the wind observations. Since the input fields reflect this inaccuracy, the divergence field derived from these winds adds noise to the forcing function. Although some of the noise could be removed by pre-filtering, we have neglected this forcing function in (18) at the present time. The effects of friction are included, however, in the determination of $u$ and $v$ from (21) and (22), respectively. The fourth term in (18) is more difficult to interpret kinematically, but can conceptually be related to the curvature in the wind field, e.g., $\partial^{2} \tilde{u} / \partial x^{2}, \partial^{2} \widetilde{u} / \partial x \partial y$, etc.

The numerical or finite-difference solution to the Euler equations is accomplished by using centered 
differences. In general, symmetric difference equations are necessary to preserve the minimization process for reasons ultimately traced to the self-adjointness of the Euler equations (Forsy the and Wasow, 1960); Stephens, 1970). Sasaki (1969) has derived algebraic computation formulas for centered difference operators to facilitate the tedious manipulations with the variational operator.

\section{Case study}

As cited earlier, the current operational analysis has no provision for including wind information in the pressure analysis. In fact, we have routinely noticed cases where the pressure analysis is obviously in error, or more properly, inconsistent with wind reports. For example, we have seen situations in mid-latitudes where strong winds have exhibited directions which are nearly perpendicular to the isobars. Because of our interest in the incorporation of wind into the pressure analysis, we have devised a test demonstrating NVA's ability to accommodate the wind field.

The region of analysis is chosen to conform with the following requirements: 1) data-rich areas on most of the boundary to anchor the analysis, 2) a sparseness of pressure data on the interior, and 3) sufficient wind observations covering the interior to demonstrate the feedback from wind to pressure.

The obvious regions satisfying the first two require' ments are oceans bounded by heavily populated continents. However, since ship traffic and island stations are the only sources of conventional data over the ocean, we generally have sparseness of wind observations. In order to satisfy the last two requirements, we have chosen an ocean region with relatively heavy traffic and have purposefully delected the pressure reports. Consequently, we get a sufficient number of wind observations to include in the analysis and, although we delete the pressure observations from the ships, we can use these reports to verify the pressure pattern obtained from the anchoring information at the coastline and the wind reports.

The analysis area is enclosed by the 0 and $90 \mathrm{~W}$ meridians and the $5 \mathrm{~S}$ and $55 \mathrm{~N}$ parallels. The continents of North America and Europe then act as data-rich boundaries to the Atlantic Ocean. The major synoptic systems are outlined in the photograph from ATS-3 at 1249 GMT and displayed in Fig. 1. From this photo and a sequence of similar photos through 1528 GMT, the National Environmental Satellite Service (NESS) derived a set of winds from the low-level cloud motion. These winds, valid at approximately $4000 \mathrm{ft}$ MSL, are depicted in Fig. 2.

Without pressure reports, the conventional analysis naturally reverts to the guess field. The operational analysis is performed every $6 \mathrm{hr}$ so that the guess is the 6-hr persistence field. Since the time scale of midlatitude systems is the order of $24-36 \mathrm{hr}$, the guess field certainly contains valuable information. However, in the case of a rapidly developing cyclone or the rapid movements of troughs and ridges, this persistence field is severely limited.

The synoptic situation off the east coast of the United States, in the vicinity of $50 \mathrm{~W}$, exemplifies noticeable eastward propagation of a trough between 0600 and 1200 GMT. This can be seen by examination of Fig. 3 which represents the conventional pressure analysis at 1200 GMT with the current observations of wind and pressure; and we reiterate, the current ship observations of pressure have been purposefully deleted. Since the conventional pressure analysis does not use the current wind, this analysis is the six-hour-old field modified by the few island reports which have been blocked to distinguish them from the ship reports.

The wind report just east of $40 \mathrm{~N}, 50 \mathrm{~W}$ would suggest an eastward movement of approximately $3^{\circ}$ and the sharp wind shift at $35 \mathrm{~N}, 50 \mathrm{~W}$ supports an intensification of the trough from the previous $6 \mathrm{hr}$. Some other noticeable discrepancies between the pressure and wind in this figure are: (i) the large cross-contour flow from low to high pressure just east of $40 \mathrm{~N}, 50 \mathrm{~W}$; (ii) the uniform pressure gradient extending southwest of $35 \mathrm{~N}, 55 \mathrm{~W}$ in the presence of winds which decrease from 40 to $15 \mathrm{kt}$ along this line; (iii) the 998 contour is clearly on the wrong side of the two ship reports in the northeast corner of the figure and the 1002 line runs between reports of 1003 and 1003.6; and (iv) the cross-contour flow into the high just south of $30 \mathrm{~N}, 50 \mathrm{~W}$.

Before comparing the NVA results with the conventional analysis, we must discuss the choice of weights $\tilde{\alpha}, \widetilde{\beta}$ and $\alpha$. In this case study, we have chosen only two sets of weights basically reflecting data-rich and datapoor points, or "land" and "sea" points, respectively. The critical value of the data density index which served to categorize the grid points was subjectively chosen. Grid points in the vicinity of clustered islands such as the Azores, with several reporting stations in close proximity, are categorized as "land" points. Conversely, there are points over South America and Africa categorized as "sea" points because observations are virtually absent.

The two sets of weights, set A ("land") and set B ("sea") are:

$$
\begin{aligned}
& \text { Set A }\left\{\begin{array}{cc}
\sigma_{p}: \sigma_{w}=1: 96, & S(p): S(u)=S(p): S(v) \approx 1: 10 \\
\text { or } & \\
\sigma_{p}: \sigma_{t}=64: 96, & S(p): S\left(u_{t}\right)=S(p): S\left(v_{t}\right) \approx 4: 5
\end{array}\right. \\
& \text { Set B }\left\{\begin{array}{cc}
\sigma_{p}: \sigma_{w}=1: 8, & S(p): S(u)=S(p): S(v) \approx 1: 3 \\
\text { or } & \\
\sigma_{p}: \sigma_{t}=8: 1, & S(p): S\left(u_{t}\right)=S(p): S\left(v_{t}\right) \approx 3: 1
\end{array}\right.
\end{aligned}
$$

where $\sigma_{p}=S^{2}(p), \quad \sigma_{w}=S^{2}(u)=S^{2}(v), \quad$ and $\sigma_{t}=S^{2}\left(u_{t}\right)$ $=S^{2}\left(v_{t}\right)$. The root-mean-square (RMS) difference between NVA and conventional wind components is indicated by $S(u)$ and $S(v)$, both expressed in knots. The RMS difference between NVA pressure and the 


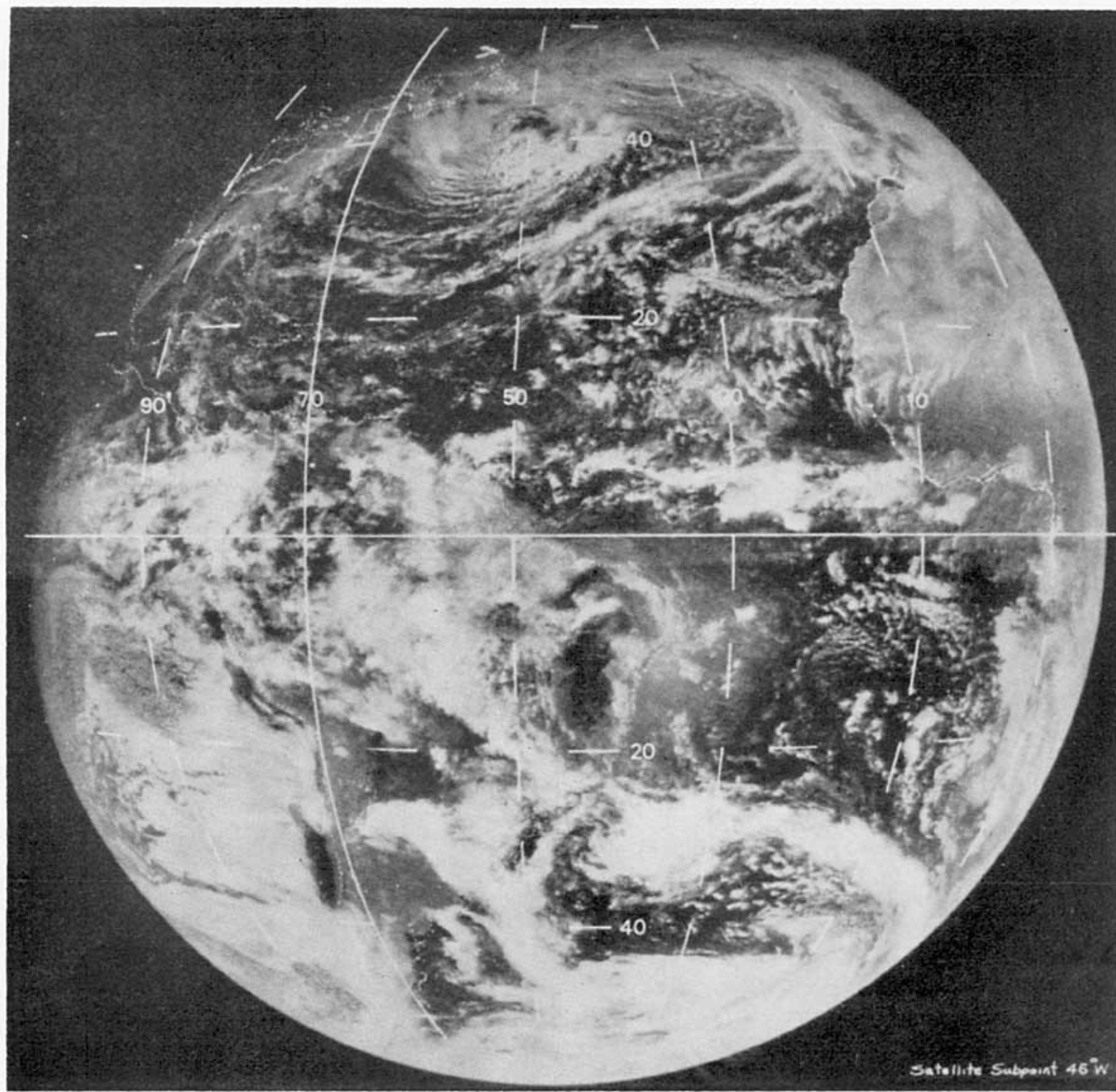

FIG. 1. ATS-3 satellite photograph taken at 1249 GMT 4 January 1971. The occluded cyclone at approximately $50 \mathrm{~N}, 30 \mathrm{~W}$ and the neighboring cyclone at $40 \mathrm{~N}, 50 \mathrm{~W}$ are vividly depicted.

conventional pressure is denoted by $S(p)$ with units in millibars. Finally, $S\left(u_{i}\right)$ and $S\left(v_{t}\right)$ are RMS local accelerations $\left(\mathrm{kt} \mathrm{hr}^{-1}\right)$ calculated from (9) and (10).

Interpreted physically, set A implies that an RMS difference of $1 \mathrm{mb}$ between NVA pressure and the input is associated with a $10 \mathrm{kt}$ RMS difference between NVA wind components and the input. These $1-\mathrm{mb}$ and $10-\mathrm{kt}$ values are associated with RMS local accelerations of approximately $1 \mathrm{kt} \mathrm{hr}^{-1}$. If the RMS difference is $0.5 \mathrm{mb}$ instead of $1 \mathrm{mb}$, a commensurate reduction is exhibited by the RMS's of wind and acceleration. Set B associates $10 \mathrm{kt}$ and $1 \mathrm{kt} \mathrm{hr}^{-1}$ values of wind and acceleration with $3 \mathrm{mb}$ for $S(p)$. The ocean area is typically represented by set $\mathrm{B}$ and permits a larger deviation between the input pressure and the NVA pressure. The ratio $r / \widetilde{\beta}$ changes by an order of magnitude from set $A$ to set $B$. For example, at $30^{\circ}$ latitude, $r / \widetilde{\beta}$ is $1 / 8$ for $A$ and $3 / 2$ for $B$, a factor of 12 . The wind information at a point in category $B$ has 12 times more effect in the solution to (18) than does the wind information at a category A point. We arrived at this weighting scheme after visually and quantitatively evaluating the patterns of pressure and wind. Sharpness of troughs and ridges, calculated local accelerations, and goodness-of-fit to observations were factors employed to make the decision.

In order to compare the NVA pressure with the conventional pressure analysis, we must also examine the input wind field $\tilde{\mathbf{V}}$, which has incorporated the current wind observations. This analysis is displayed in 


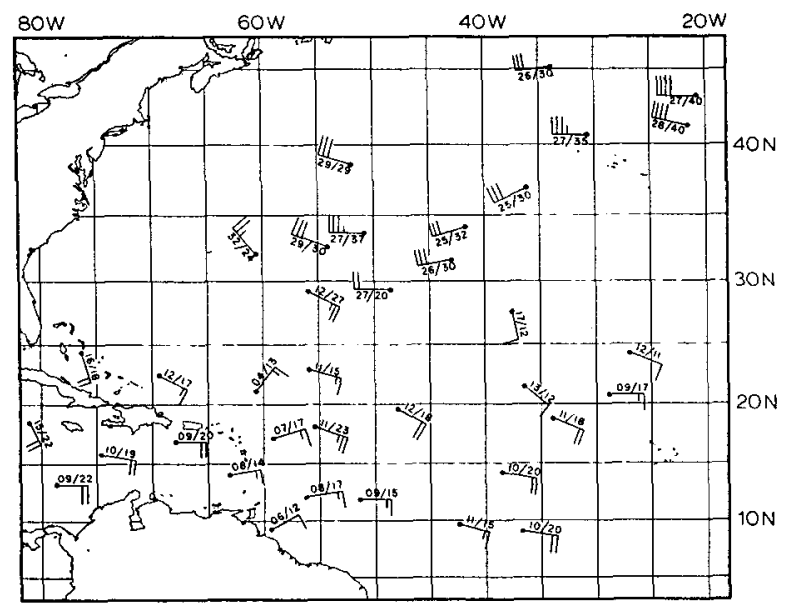

FIG. 2. Low-level winds ( $\sim 4000 \mathrm{ft} \mathrm{MSL})$ derived from cloud trajectories at the National Environmental Satellite Service.

Fig. 4 and for convenience of reference, we have superimposed the input pressure analysis $\tilde{p}$.

Fig. 5 is the NVA pressure analysis at 1200 GMT with the observations superimposed. Only the blocked pressure observations, island and continental stations, have been used. Now the wind shift east of $50 \mathrm{~W}$ and between 30 and $40 \mathrm{~N}$ which is apparent from the input winds (Fig. 4) has been captured by the NVA pressure pattern. Also, another trough extending SW from $40 \mathrm{~N}$, $50 \mathrm{~W}$ is apparent on the NVA pressure again supported by the input wind field. The anticyclonic flow just south of $30 \mathrm{~N}$, 50W in Fig. 4 causes a noticeable shifting of the 1018 line.

Some other improvements resulting from the NVA adjustment are the orientation of the 1002 line just north of $40 \mathrm{~N}, 50 \mathrm{~W}$, the position of the 998 line relative to the ship reports in the northeast corner, and the

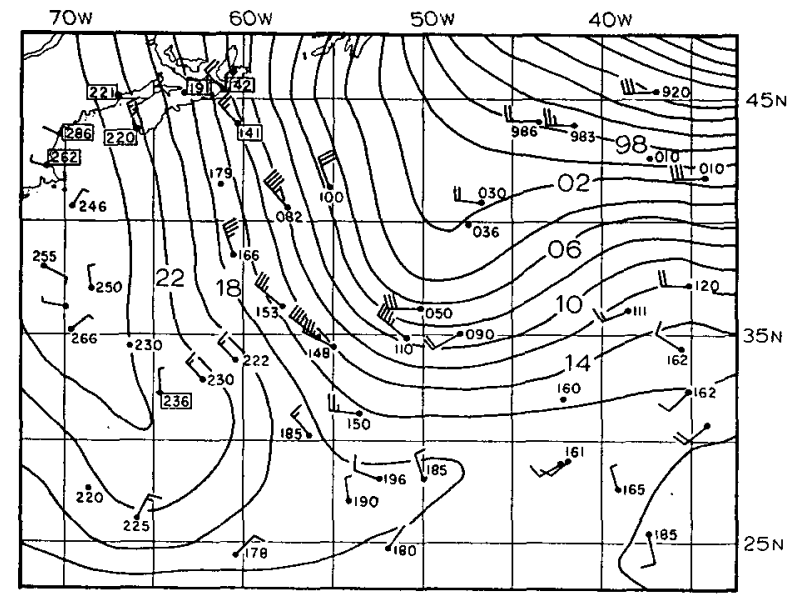

FIG. 3. Conventional analysis of sea level pressure at 1200 GMT 4 January 1971 . Only those pressures that are blocked, i.e., reports from continental or island stations, have been used. Wind observations are superimposed.

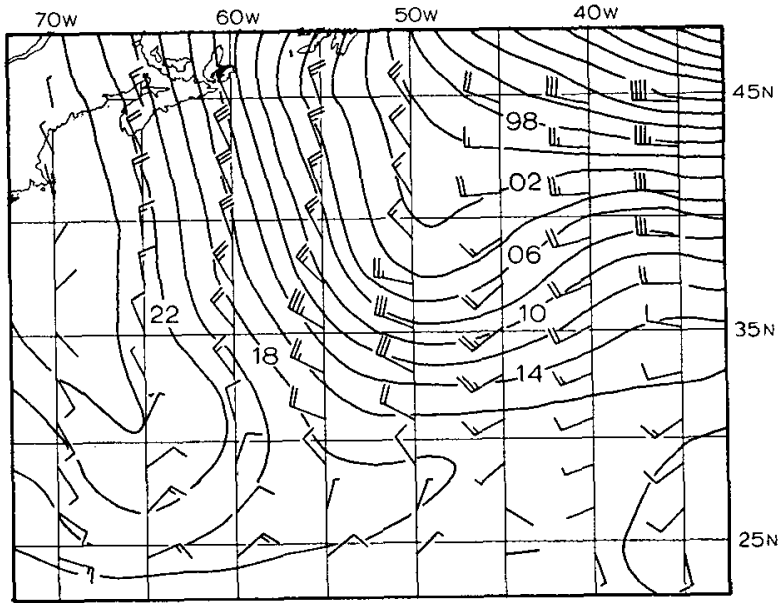

FIG. 4. Conventional wind analysis at 1200 GMT 4 January 1971 plotted over the conventional pressure analysis. Alternate columns of gridded wind barbs have been purposefully deleted to prevent overcrowding.

strengthening of the pressure gradient in the vicinity of the strong winds NE of $35 \mathrm{~N}, 60 \mathrm{~W}$.

The NVA wind pattern is displayed in Fig. 6. Again, in analogy with the input fields, the NVA pressure pattern has been superimposed. To enhance the differences between the NVA wind and the input, the vector difference, $\mathbf{V}-\tilde{\mathbf{V}}$, is shown in Fig. 7 and this vector is superimposed on the NVA pressure. This difference field indicates that the NVA wind exhibits a more pronounced cross-contour flow from high toward low pressure. Remembering that the input winds are essentially geostrophic because of the first guess, this result was anticipated and is attributable to the inclusion of friction in the dynamics.

There is evidence that the northwesterly component behind (to the west) the trough has been reduced by NVA. Examination of these input winds in Fig. 4 shows

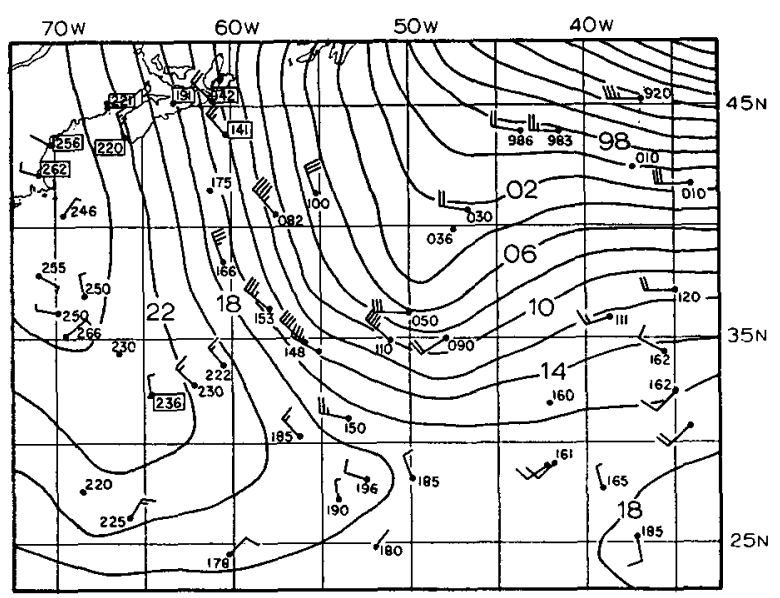

FIG. 5. NVA pressure at 1200 GMT 4 January 1971 . Only those pressures that are blocked, i.e., reports from continental or island stations, have been used. Wind observations are superimposed. 


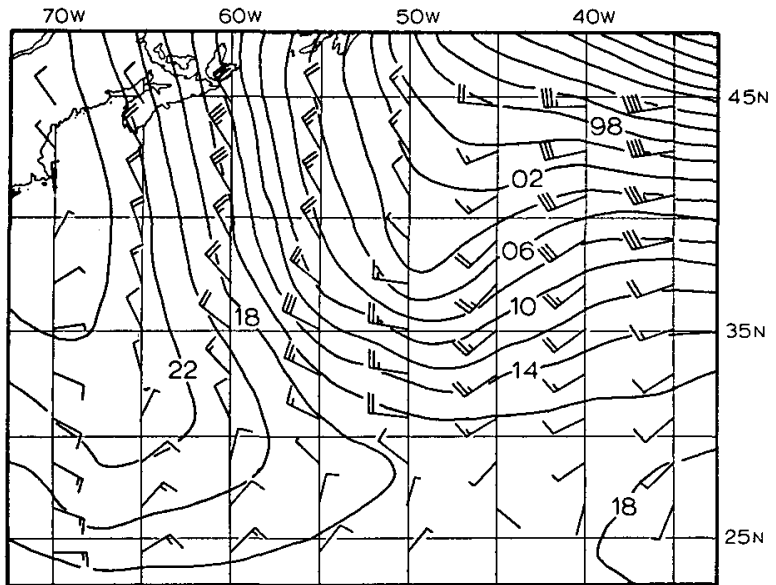

FIG. 6. NVA wind at 1200 GMT 4 January 1971 plotted over the NVA pressure. Alternate columns of gridded wind barbs have been purposefully deleted to prevent overcrowding.

cyclonic flow and, accordingly, a geostrophic wind tends to overestimate the speed. The nonlinear terms $\widetilde{A}$ and $\widetilde{B}$ appearing in (21) and (22) are responsible for this

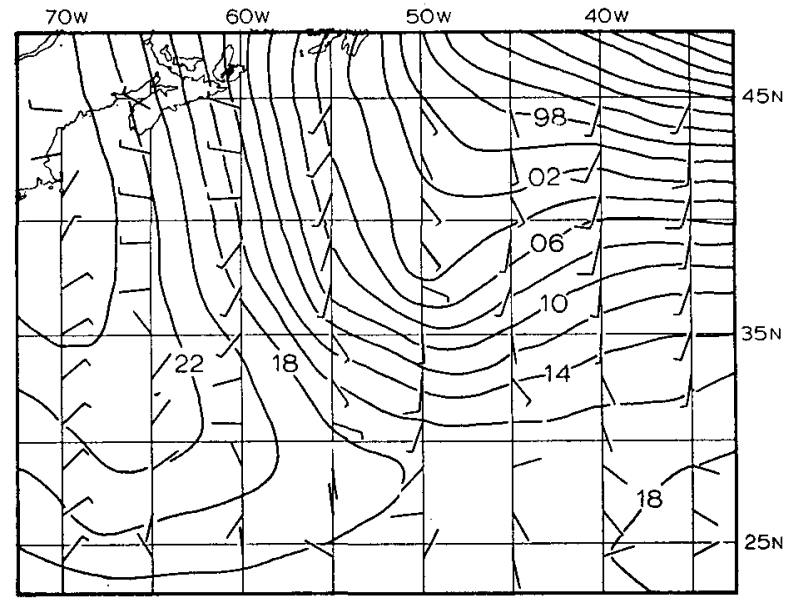

FIG. 7. Vector difference between the NVA wind and the conventional wind, $\mathbf{V}-\tilde{\mathbf{V}}$. This vector field is superimposed on the NVA pressure pattern.

desired reduction of wind speed. The anticyclonic flow east of the trough that is apparent in the input winds has the opposite effect. Namely, the geostrophic wind

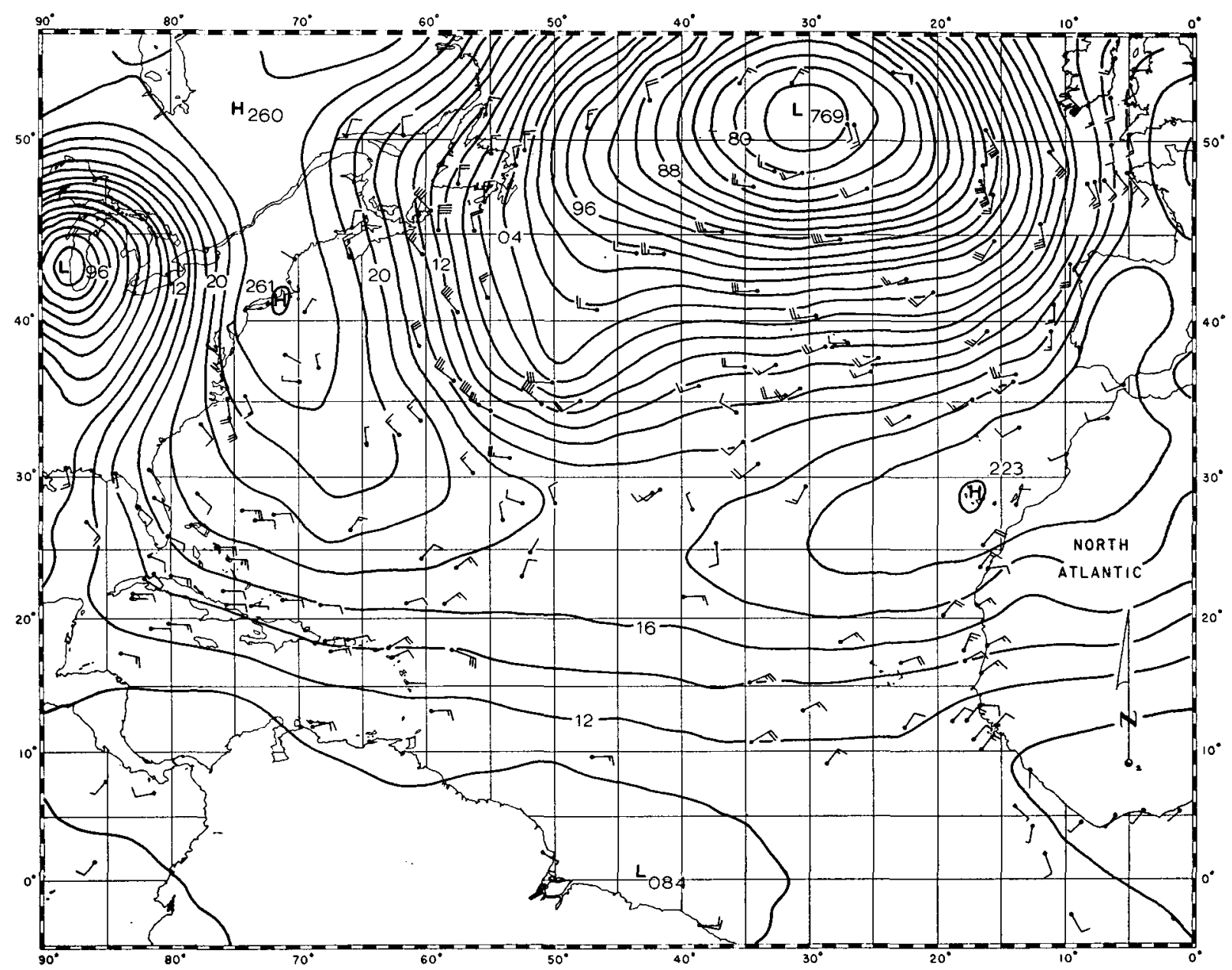

FIG. 8. NVA pressure analysis at 1200 GMT 4 January 1971, with wind observations superimposed. 
underestimates the wind speed for anticyclonic systems and the input field reflects this fact. Again, NVA increases the wind speed by incorporating the nonlinear terms.

An expanded view of the NVA pressure with superimposed wind observations is shown in Fig. 8. The analysis showed skill in creating a slight inverted trough by the West Indies ( $60 \mathrm{~W}$ between $15 \mathrm{~N}$ and $20 \mathrm{~N}$ ). This is supported by the wind observations but the input analysis showed essentially horizontal isobars in this area. A simple analytical study of NVA's ability to accommodate this type of situation is discussed in the Appendix.

\section{Verification}

The quantitative evaluation of the NVA results consists of two primary parts: RMS differences between NVA and conventional analyses and comparison of ratios of these differences to the hypothetical ratios, and RMS errors (RMSE) between the observations and each analysis. The results in Table 1 indicate that NVA pressure deviated from the input at set B ("sea") points by nearly $1 \mathrm{mb}$ in an RMS sense. The deviation at set A ("land") points is significantly smaller as expected. Although it is difficult to specify a scale value of local acceleration for the entire analysis region, the RMS values $S\left(u_{t}\right)$ and $S\left(v_{t}\right)$ seem reasonable in each category. These values scale an order of magnitude smaller than the Coriolis and pressure gradient forces of mid-latitudes.

The calculated and hypothetical ratios of the RMS differences are displayed in Table 2. There is some discrepancy between the hypothetical and calculated values, but this can be anticipated for the following reasons: 1 ) the solutions to the Euler equations couple the grid points in categories $\mathrm{A}$ and $\mathrm{B}$, i.e., set $\mathrm{A}$ grid points are influenced by the information at set $B$ points and vice versa (Only for the case of uniform weighting over the entire grid will this effect be removed.);2) the finite-difference scheme used for numerical integrations of the Euler equations acts as a filter and naturally creates differences between the analytic and numerical solutions; and 3) the forcing function for one of the Euler equations $[\mathrm{Eq}$. (18)] is incomplete because we neglected the term involving divergence.

The RMSE between each analysis and the observations is shown in Table 3. The wind error indicated in

TABLE 1. Root-mean-square differences as a function of grid point category.

\begin{tabular}{cccccc}
\hline Category & $\begin{array}{c}S(u) \\
(\mathrm{kt})\end{array}$ & $\begin{array}{c}S(v) \\
(\mathrm{kt})\end{array}$ & $\begin{array}{c}S(p) \\
(\mathrm{mb})\end{array}$ & $\begin{array}{c}S\left(u_{t}\right) \\
\left(\mathrm{kt} \mathrm{hr}^{-1}\right)\end{array}$ & $\begin{array}{c}S\left(v_{t}\right) \\
\left(\mathrm{kt} \mathrm{hr}^{-1}\right)\end{array}$ \\
\hline $\begin{array}{c}\text { Set A } \\
\text { (619 grid points) }\end{array}$ & 3.9 & 4.8 & 0.42 & 0.28 & 0.26 \\
$\begin{array}{c}\text { Set B } \\
\text { (483 grid points) }\end{array}$ & 2.9 & 3.5 & 0.91 & 0.26 & 0.19 \\
\hline
\end{tabular}

TABLE 2. Ratios of the root-mean-square difference as a function of category. The numbers in parentheses represent the hypothetical ratios based on the weighting in the analysis scheme.

\begin{tabular}{ccccc}
\hline \hline Category & $\frac{S(p)}{S(u)}$ & $\frac{S(p)}{S(v)}$ & $\frac{S(p)}{S(u t)}$ & $\frac{S(p)}{S\left(v_{t}\right)}$ \\
\hline Set A & 0.093 & 0.110 & 1.5 & 1.6 \\
(619 grid points) & $(0.102)$ & $(0.102)$ & $(0.82)$ & $(0.82)$ \\
Set B & 0.30 & 0.23 & 3.1 & 4.8 \\
(483 grid points) & $(0.35)$ & $(0.35)$ & $(2.8)$ & $(2.8)$ \\
\hline
\end{tabular}

TABLE 3. Root-mean-square error between observations and the analyses interpolated to the location of the observations. Ship observations of pressure have been included in this verification.

\begin{tabular}{llcccc}
\hline \multicolumn{1}{c}{ Region } & & $\begin{array}{c}\mathrm{V} \\
(\mathrm{kt})\end{array}$ & $\begin{array}{c}\tilde{\mathrm{v}} \\
(\mathrm{kt})\end{array}$ & $\begin{array}{c}p \\
(\mathrm{mb})\end{array}$ & $\begin{array}{c}\tilde{p} \\
(\mathrm{mb})\end{array}$ \\
\hline $\begin{array}{l}5 \mathrm{~S}-55 \mathrm{~N} \\
0-90 \mathrm{~W}\end{array}$ & $(\mathrm{I})$ & 7.7 & 5.0 & 1.0 & 0.91 \\
$\begin{array}{l}30 \mathrm{~N}-45 \mathrm{~N} \\
40 \mathrm{~W}-55 \mathrm{~W}\end{array}$ & (II) & 7.8 & 5.2 & 0.76 & 1.3 \\
\hline
\end{tabular}

this table is the magnitude of the vector difference between observed and analyzed. Bessel's interpolation scheme was used to find the analyzed value at the observation point.

The NVA analysis exhibits a larger RMSE in both wind and pressure in region $I$, the entire analysis region. The conventional analysis has "drawn" to the observations without regard for dynamical constraints. Since the observations contain various scales, some inconsistent with the model constraints, this result can be expected. Yanii (1964) obtained this same result when he modified the SCM pressure by a statistical-dynamical model which assumed a balance between Coriolis, pressure gradient and frictional forces. The idea was formulated by Masuda and Arakawa (1962).

It is interesting to note that in the case of the welldefined wind system (pronounced cyclonic flow) in region II, the RMSE for pressure was reduced by the NVA scheme. Here the NVA pressure obviously benefited from the wind information contained in the forcing function of (18). In effect, the curvature of the isobars could be coupled with the vorticity, whereas the conventional analysis could not incorporate this information.

\section{Concluding remarks}

Based on the encouraging results of the case study, the NVA scheme has been programmed for operational use at FNWC. Routine execution of the operational version began on 11 November 1971. This analysis scheme requires an additional $2 \mathrm{~min}$ of CPU (Central Processor Unit) time on the Control Data 6500 when run in tandem with the SCM. The Liebmann iteration 
method is used to solve (18) and requires about 20 iterations to obtain an accuracy of $0.1 \mathrm{mb}$.

The operational version has exhibited many of the features displayed in this case study. In particular, better definition of short-wave troughs and ridges has resulted from incorporation of the wind field into the pressure analysis. Also, the time continuity from one map analysis to the next, a 6-hr interval, has noticeably improved.

Despite the encouraging results, we have a bias in our weighting scheme which was uncovered by one of the reviewers of this paper. In formulating the functional [Eq. (6)], the Jacobian of the transformation from earth to map coordinates was neglected. Consequently, we have inadvertantly given equal weighting to equal map areas and not equal earth areas. Our present analysis has excess weighting (area average sense) above $22.5 \mathrm{~N}$ and below $22.5 \mathrm{~S}$, and correspondingly, too small a weighting within the band. The Euler equations are being modified in the operational program to overcome this bias.

Besides modifying the program to account for the weighting bias, an iteration scheme is being tested which gives a better approximation to the nonlinear terms. After the solutions $p, u$ and $v$ are found from (18), (21) and (22), respectively, the $u$ and $v$ solutions are used to re-calculate the nonlinear terms, $\widetilde{A}$ and $\widetilde{B}$. Once again, (18), (21) and (22) are solved with the improved estimates for $\widetilde{A}$ and $\widetilde{B}$.

A long-range plan involves the direct inclusion of the tendency of observations into the analysis. This can be accomplished by using an NVA formulation (so-called "weak constraint" form) discussed by Sasaki (1970b). The Euler equations then contain time derivatives and can directly assimilate off-time and asynoptic observations. The derivation of the governing Euler equations for the dynamical constraints imposed in this study is straightforward. However, the necessary increase in both computer time and memory to accommodate this extension is prohibitive at the present time.

\section{APPENDIX}

\section{Analytical Example of Wind and Pressure Interaction in the Variational Scheme}

This simplified mathematical example is patterned after the event appearing on the extended sea level chart (Fig. 8), located near the West Indies (near 20N, $60 \mathrm{~W}$ ). In this region, the following evidence supports the existence of an inverted short-wave trough: (i) the definite cyclonic character shown by the wind observations, especially the stronger winds of 20 and $30 \mathrm{kt}$; and (ii) the wind analysis derived at the National Environmental Satellite Service (NESS) by following low-level clouds (Fig. 2). These satellite-derived winds were obtained from cumulus cloud movements calculated from the successive ATS-3 cloud photographs between 1200 and 1500 GMT on 4 January.

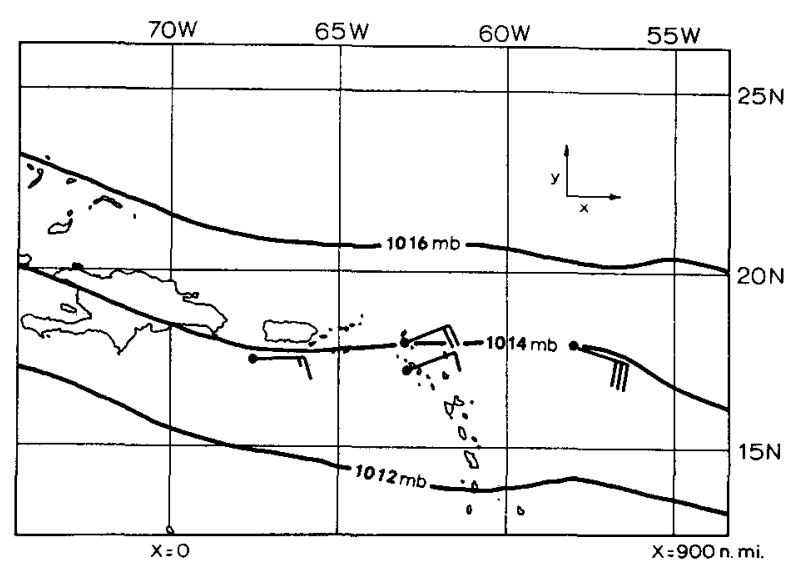

FIG. 9. Enlarged view of the NVA pressure pattern in the vicinity of the West Indies with wind observations superimposed.

Since the conventional pressure analysis disregards the wind information and since the pressure reports have been purposefully deleted over the oceans, persistence (the six-hour-old pressure pattern) is used. There was no indication of troughing on this input pressure pattern. The isobars were virtually straight and oriented east-west.

A blow-up of the NVA pattern is shown in Fig. 9 and the curvature of the isobars in response to the winds is evident. In order to demonstrate how the NVA scheme accomplishes this, the following information is assumed to be available from the conventional pressure and wind analysis:

$$
\begin{gathered}
\tilde{p}=P_{0}+\left(\frac{d p}{d y}\right)_{0} \cdot y, \frac{\partial \tilde{p}}{\partial x}=0, \\
\tilde{u}=-U-u^{*} \cos k x, \\
\tilde{v}=-v^{*} \sin k x,
\end{gathered}
$$

where

$$
k=\frac{2 \pi}{L}
$$

$\begin{array}{cl}\left(\frac{d p}{d y}\right)_{0}, \begin{array}{l}\text { pressure gradient from } \\ \text { conventional analysis }\end{array} & : 4 \mathrm{mb}(600 \mathrm{n} \mathrm{mi})^{-1} \\ P_{0}, \text { reference pressure } & : 1014 \mathrm{mb}\end{array}$

For purposes of argument, we assume $y=0$ coincides with the latitude circle which represents the mean position of the 1014-mb contour in Fig. 9. Using (A1)- 


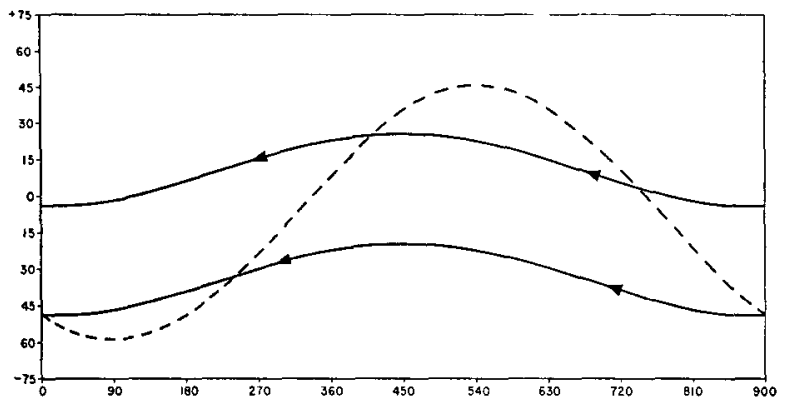

FIG. 10. The displacement of the 1014-mb contour resulting from the NVA scheme. All distances are in nautical miles. Streamlines of the input wind $\tilde{\mathbf{V}}$ are indicated by the solid sinusoids.

(A3) as input to NVA, the solution to Eq. (18) is

$$
\begin{array}{r}
p=\tilde{p}+\frac{\left(u^{*} k\right)^{2}}{\rho(\tilde{\beta} / r)}+\frac{\rho f v^{*} k+U u^{*} k^{2} \rho}{k^{2}+\rho^{2}(\tilde{\beta} / r)} \cos (k x) \\
+\frac{\kappa u^{*} k \rho}{k^{2}+\rho^{2}(\widetilde{\beta} / r)} \sin (k x) .
\end{array}
$$

It is clear that the ratio $\widetilde{\beta} / r$ plays an important role in bringing the wind information into the pressure pattern. As $\tilde{\beta} / r$ increases, the winds are less effective in modifying the pressure. In fact, as $\tilde{\beta} / r \rightarrow \infty$, then the NVA solution reverts to the input field $\tilde{p}$ as would be expected.

Using the weights specified in set $B$, the NVA pressure pattern and the streamlines of the input winds are shown in Fig. 10.

The pressure pattern depicts the inverted trough and exhibits a phase shift relative to the streamline which suggests a net cross-contour flow from high toward low pressure. To estimate this cross-contour flow, the flux across a line of constant pressure is found to be

$$
\int_{\Gamma} \tilde{\mathbf{V}} \cdot \hat{\mathbf{n}} d s=\frac{\left(k u^{*}\right)^{2} \kappa \rho L}{2\left[k^{2}+\rho^{2}(\tilde{\beta} / r)\right](d p / d y)_{0}},
$$

where $d s$ is an incremental segment of the analytical NVA isobar (denoted by $\Gamma$ ) between 55 and $70 \mathrm{~W}$, and the unit vector $\hat{\mathbf{n}}$ is normal to the isobar and directed from high to low pressure. Using the scale values mentioned earlier, the net flux across the isobar is $\sim 10^{2}$ (n mi $)^{2} \mathrm{hr}^{-1}$ or, equivalently, the average cross-contour velocity component is $\sim 0.1 \mathrm{kt}$. As expected, the net flux is directly proportional to the frictional parameter $\kappa$.

Acknowledgments. The guidance from JAM editor, Prof. Edward S. Epstein, and the scrutiny of the reviewers has been extremely valuable. The continued dialogue with Prof. Russell Elsberry and two of his graduate students, Charles Steinbruck and Roger Langland, of the Naval Postgraduate School, has contributed significantly. Also, the encouragement from both Profs. Yoshikazu Sasaki and Bo Döös has been appreciated. Finally, the careful typing of the manuscript by Mrs. Judy Scott is gratefully acknowledged.

\section{REFERENCES}

Bergthórsson, P., and B. Döös, 1955: Numerical weather map analysis. Tellus, 7, 329-340.

Charney, J. G., 1955: The use of the primitive equations of motion in numerical prediction. Tellus, 7, 22-26.

Cramér, H., 1955: The Elements of Probability Theory. New York, Wiley, 281 pp. (see p. 235).

Cressman, G. P., 1959: An operational objective analysis system. Mon. Wea. Rev., 94, 367-374.

Forsythe, G. E., and W. R. Wasow, 1960: Finite-Difference Methods for Partial Differential Equations. New York, Wiley, $444 \mathrm{pp}$.

Gandin, L. S., 1965: Objective Analysis of Meteorological Fields. Israel Program for Scientific Translations. Also Tech, Rept. TT65-5007, Clearinghouse for Federal Scientific and Technical Information, $242 \mathrm{pp}$.

Grayson, T. H., 1971 : Global band sea level pressure and surface wind analysis. Tech. Note 71-3, Fleet Numerical Weather Central, Monterey, Calif., 22 pp.

Hess, S., 1959: Introduclion to Theorelical Meleorology. New York, Holt, 362 pp. (see p. 179).

Houghton, D., and W. Washington, 1969: On global initialization of the primitive equations: Part I. J. Appl. Meteor., 8, 726-737.

Hubert, W. E., and B. R. Mendenhall, 1970: The FNWC singular sea swell model. Tech. Note 59, Fleet Numerical Weather Central, Monterey, Calif., $29 \mathrm{pp}$.

Kung, E. C., 1963: Climatology of aerodynamic roughness parameter and energy dissipation in the planetary boundary layer of the northern hemisphere. Annual Report, Contract DA-36-039-AMC-00878, Dept. of Meteorology, University of Wisconsin, 37-96.

Lanczos, C., 1970: The Variational Principles of Mechanics, 4th ed. University of Toronto Press, Math. Expositions No. 4, 375 pp. (see p. 70).

Lettau, Heinz, H., 1959: Wind profile, surface stress and geostrophic drag coefficients in the atmospheric surface layers. Advance in Geophysics, Vol. 6, New York, Pergamon Press, 241-257.

Lewis, J. M., 1971 : Variational subsynoptic analysis with applications to severe local storms. Mon. Wea. Rev., 99, 786-795.

Maine, R., and R. S. Seaman, 1967: Development for an operational automatic weather analysis system in the Australian Region. A ustralian Meleor. Mag., 13-31.

Masuda, Y., and A. Arakawa, 1962: On the objective analysis for surface and upper level maps. Proc. Intern. Symp. Nutmerical Weather Prediction Tokyo, 1960, Meteor. Soc. Japan, $55-66$.

Sasaki, Y., 1958: An objective analysis based on the variational method. J. Meteor. Soc. Japan, 36, 77-88.

- 1969 : Proposed inclusion of time variation terms, observational and theoretical, in numerical variational objective analysis. J. Meteor. Soc. Japan, 47, 115-124.

- $1970 \mathrm{a}$ : Some basic formalisms in numerical variational analysis. Mon. Wea. Rev., 98, 875-883.

$\longrightarrow, 1970 \mathrm{~b}$ : Numerical variational analysis with weak constraint and application to surface analysis of severe storm gust. Mon. Wea. Rev., 98, 899-910.

Stephens, J. J., 1966: A variational approach to numerical weather analysis and prediction. Rept. No. 3, Atmos. Sci. Group, University of Texas, Austin, 243 pp.

,- 1970 : Variational initialization with the balance equation. J. Appl. Meteor., 9, 732-739.

Yanii, M., 1964: An experimental objective analysis in the tropics. Tech. Paper No. 22, Dept. of Atmos. Sci., Colorado State University $21 \mathrm{pp}$. 